\title{
What predicts the severity of alcohol use and related problems among young people presenting to emergency department or crisis support care?
}

\author{
Catherine A Quinn ${ }^{*}$, Leanne Hides \\ From INEBRIA 12th Congress, \\ Atlanda, GA, USA. 24-25 September 2015
}

\section{Background}

Harmful and hazardous drinking is endemic among young people. In recent decades, there have been dramatic increases in the number of young people presenting to emergency departments with alcohol-related injuries and illnesses (inc. intoxication). Yet, little is known about what predicts the severity of alcohol use and related problems in these traditionally non-treatment seeking samples of young people.

This paper identifies what treatment orientated factors, including readiness to change, self-efficacy to reduce heavy drinking and coping strategies, relate to alcohol use and -related problems in young people presenting to an emergency department or crisis support care.

\section{Material and methods}

Participants were 331 (52\% female) young Australians aged 16-25 years (Mage $=20.3$ years), who had come into contact with an emergency department $(17.2 \%)$ or crisis support care (82.8\%) with alcohol related injuries or illnesses and were referred to a brief alcohol intervention trial.

\section{Results}

The average number of standard drinks consumed on the night of contact was $14.72(\mathrm{SD}=9.04)$. A high proportion had not used illicit substances $(78.2 \%)$ on the night of contact, had low psychological distress (61\%), were precontemplative (90.8\%), and had high confidence in their ability to stop themselves drinking heavily in most circumstances, except social situations. Regression analyses revealed that confidence to stop drinking was inversely related to alcohol problems and the number of drinks consumed on the night of contact. Readiness to change and emotion-oriented coping were positively related to alcohol-related problems.

\section{Conclusions}

Despite engaging in risky alcohol use and experiencing alcohol-related problems, this sample of young people were quite confident in their ability to control their drinking. Potential targets for brief interventions will be discussed.

\begin{abstract}
Acknowledgements
Participants for this presentation were recruited for the Quikfix Randomized Control Trial. Quikfix is funded by the National Health and Medical Research Council (NHMRC) (APP1044046). The Chief Investigators are Leanne Hides, David Kavanagh, Mark Daglish, Susan Cotton, Jason Connor, Jan Barendregt, Ross Young and Angela White. Associate investigators are Davina Sanders and Lance Mergard. Leanne Hides is supported by an Australian Research Council Future Fellowship. We would like to acknowledge the assistance of Chaplain Watch and Royal Brisbane Department of Emergency Medicine and the young people who have agreed to participate in this research.
\end{abstract}

Published: 24 September 2015

doi:10.1186/1940-0640-10-S2-033

Cite this article as: Quinn and Hides: What predicts the severity of alcohol use and related problems among young people presenting to emergency department or crisis support care? Addiction Science \& Clinical Practice 2015 10(Suppl 2):O33.

\footnotetext{
* Correspondence: c6.quinn@qut.edu.au

Centre for Youth Substance Abuse Research, School of Psychology \&

Counselling, Institute of Health \& Biomedical Innovation, Queensland University of Technology, Brisbane, Australia
} 